\title{
Some Aspects of the Natural Glaciation Processes in Relatively Warm Maritime Clouds
}

\author{
By A. Ono \\ Meteorological Research Institute, Tokyo, Japan \\ (Manuscript received 7 May, 1971)
}

\begin{abstract}
Evidence is presented that small regular ice crystals found in maritime cumulus clouds with summit temperature warmer than $-10 \mathrm{C}$ were formed in clouds near the sampling level. Their concentrations were far larger than those usually observed for natural ice nuclei, so that it is supposed that they were the secondary products and the primary ice crystals growing on natural ice nuclei could be multiplied many times in the course of the formation of small regular ice crystals. Their sizes, concentrations and microphysical conditions of occurrence support the hypothesis that they were formed when ice fragments were thrown off from water drops freezing after accreting on ice crystals.
\end{abstract}

\section{Introduction}

Observations of ice crystals in natural clouds made by various groups have shown their concentrations to be 3 or 4 orders of magnitude larger than the numbers which form in a conventional cloud chamber at the cloud summit temperature, suggesting the existence of some very active ice crystal enhancement mechanism.

Because there is at present a very large amount of operational cloud-seeding carried out in various parts of the world, an understanding of this crystal enhancement phenomenon is of great importance. If a cloud is capable of complete glaciation in the presence of natural ice nuclei only it is possible that additional ice nuclei injected artificially would inhibit precipitation rather than stimulate it.

Various authors have proposed such ideas as seeding by ice crystals formed at higher levels, the fracture of structually fragile forms during growth by condensation or riming, the shattering or fragmentation of freezing water drops, or accumulation zones for ice crystals whose fall velocities balance with the updraft.

Unless we have evidence of the conditions existing in the clouds before the crystals are large or numerous enough to alter cloud con- ditions substantially, we have no hope of deciding which if any of these mechanisms is the real one. For example, in our observations of the last two winters (Mossop and Ono, 1969) we examined conditions in many clouds, but most of them were in too advanced a stage of glaciation for providing relevant informations on how the glaciation had arisen. Therefore, in cloud sampling experiments made off the coast of Tasmania in May 1968, we concentrated on flying through maritime cumulus clouds where cloud summit temperatures were warmer than $-10 \mathrm{C}$.

On many occasions we succeeded in sampling clouds which were in the developing stage of glaciation and the microphysical conditions giving rise to it were still present. One of the striking features of the clouds was that many very small regular ice crystals were observed during passage through the clouds as well as large ice crystals, rimed ice crystals, graupel ice particles and many supercooled water droplets fairly large in size. Providing that there was still sufficient liquid water in the cloud, we can assume that if they came from the cloud, then they had only recently formed near the sampling level, for they would grow rapidly in the presence of liquid water.

The presence of these very small regular 
ice crystals in some clouds interested us in their origins: whether or not these crystals arise on ice nuclei in the atmosphere.

The measured concentrations of very small ice crystals were 2 or 3 orders of magnitude larger than those of ice nuclei activated at the temperatures of the sampling level. A necessary conclusion, therefore, seems to be that these very small ice crystals could not have originated from the ice nuclei present in the atmosphere, suggesting that they may be the secondary products and the primary ice crystals may be multiplied many times in the course of the formation of very small regular ice crystals.

The aim of the present paper is to examine in detail the nature and origin of these small ice crystals in relation to the ice crystal multiplication and ascertain the microphysical conditions existing at the time.

So far the observation of small regular ice crystals was reported by Koenig (1968), who observed on a mountain top the presence of small regular ice crystals in clouds of relatively warm summit and suggested that they might be the secondary products due to the crystal multiplication processes. However, no detailed studies have been done concerning the concentration and nature of these small ice crystals.

\section{Source of data}

The present studies are based on the cloud particles sampling experiments carried out in May 1968 off the coast of Tasmania using an instrumented DC-3 aircraft.

Tasmania lying well to the south of mainland Australia is usually located in a purely maritime air mass. The majority of clouds studied were formed in moderately unstable air shortly after the passage of a cold front. No high cloud was present except on 25 May (cirrus).

Sampling was carried out during horizontal flight through a maritime cumulus cloud at an intended level of $300 \mathrm{~m}$ below the summit. Cloud dimensions and temperatures are summarized in Table 1.

Measurements of the concentrations and the nature of ice crystals were obtained from the replicas of ice crystals which were collected using a decelerating tube to slow the crystals down before impacting them on a
Table 1. Cloud dimensions and tepmeratures

\begin{tabular}{l|l}
\hline \multicolumn{1}{c|}{ Parameter } & Range \\
\hline Height of cloud $(\mathrm{km})$ & 0.8 to 1.1 \\
Temperature of cloud base $\left({ }^{\circ} \mathrm{C}\right)$ & +5 to 0 \\
Depth of cloud (km) & 1.1 to 2.0 \\
Cloud summit temperature $\left({ }^{\circ} \mathrm{C}\right)$ & -4 to -10 \\
$\begin{array}{l}\text { Cloud width at penetration level } \\
(\mathrm{km})\end{array}$ & 0.4 to 9.0 \\
\hline
\end{tabular}

glass slide, thus reducing the probabilities of their being shattered on collection. Further details of the method of crystal sampling and its performance are given by Mossop et al. (1970).

\section{Physical properties of ice crystals observed in clouds}

The size distributions of ice crystals observed in clouds are plotted in Fig. 1 (a, b, c, and d). Their sizes range from 10 to $500 \mu$ along the major axis, indicating that crystals of very different growth times were sampled simultaneously. The "accumulation zone" hypothesis for explaining large ice crystal concentrations requires that many of the crystals should have about the same size balanced by updraft velocity. The wide size distribution of ice crystals shown in Fig. 1 seems to rule out this mechanism. The hatched area in the figures is the region where rimed ice crystals were observed.

In earlier publications (Ono, 1969 and 1970 ) it was shown that for columnar ice crystals, growth along the minor axis ceases almost completely after it reaches a certain length which depends on the growing temperatures. Fig. 1 confirms this.

The growth mode of columnar ice crystals has been investigated in about 40 clouds over the temperature range -3 to $-10 \mathrm{C}$ with results shown in Fig. 2 (after Ono, 1970). The boundary for needle-type ice crystals observed at temperatures between -4 and $-6 \mathrm{C}$ is marked with chain lines, for sheathtype ice crystals observed between -6 and $-8 \mathrm{C}$ with solid lines and for the solid/ hollow-type ice crystals observed at -3.5 and -8 to $-9.5 \mathrm{C}$ with broken lines. Updraft and falling motion of ice crystals in clouds undoubtedly allow us at a particular level to sample crystals from either lower or higher level, which may be the reason for the super- 

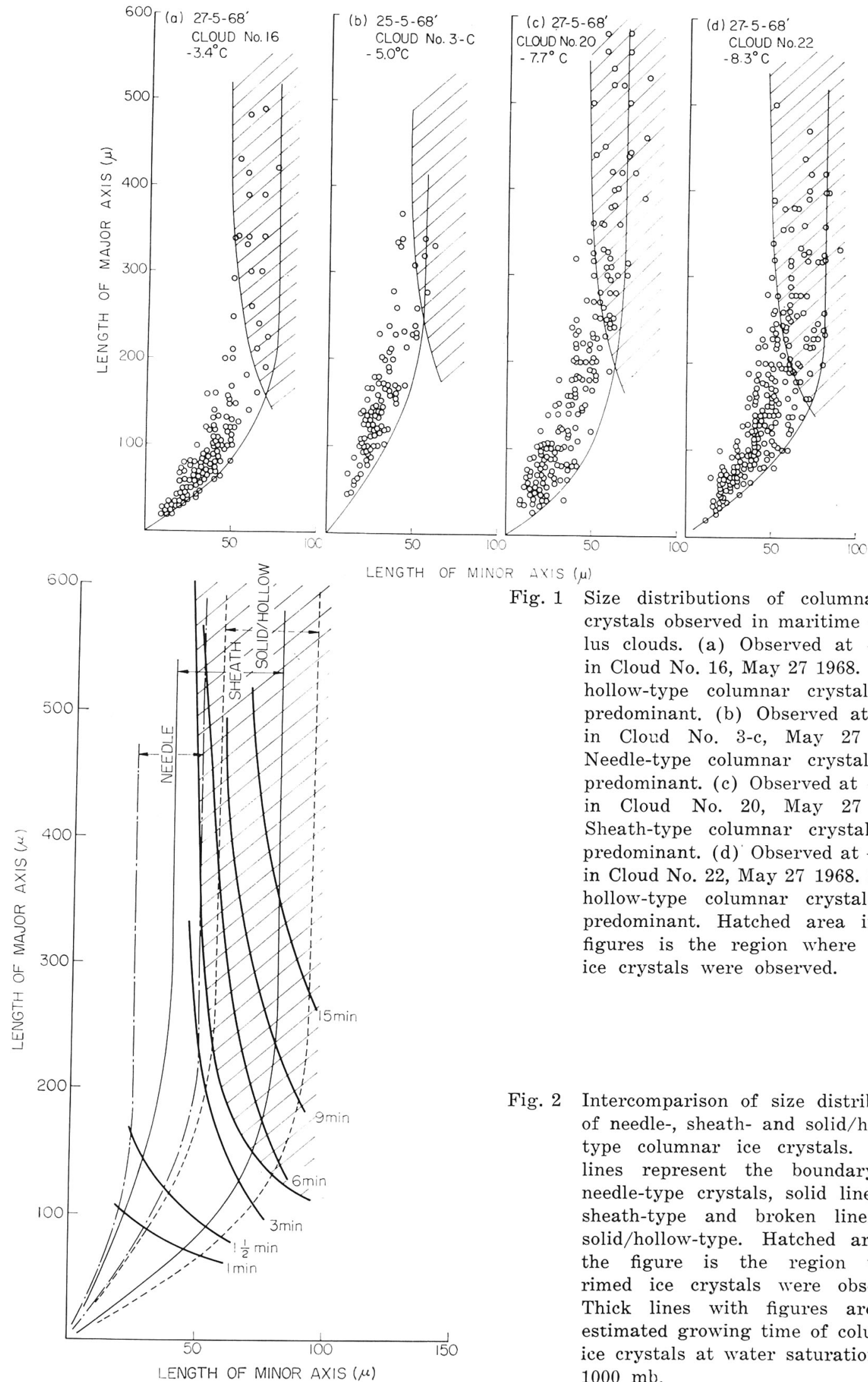

Fig. 1 Size distributions of columnar ice crystals observed in maritime cumulus clouds. (a) Observed at $-3.4 \mathrm{C}$ in Cloud No. 16, May 27 1968. Solid/ hollow-type columnar crystals are predominant. (b) Observed at $-5 \mathrm{C}$ in Cloud No. 3-c, May 271968. Needle-type columnar crystals are predominant. (c) Observed at $-7.7 \mathrm{C}$ in Cloud No. 20, May 271968. Sheath-type columnar crystals are predominant. (d) Observed at $-8.3 \mathrm{C}$ in Cloud No. 22, May 27 1968. Solid/ hollow-type columnar crystals are predominant. Hatched area in the figures is the region where rimed ice crystals were observed.

Fig. 2 Intercomparison of size distribution of needle-, sheath- and solid/hollowtype columnar ice crystals. Chain lines represent the boundary for needle-type crystals, solid lines for sheath-type and broken lines for solid/hollow-type. Hatched area in the figure is the region where rimed ice crystals were observed. Thick lines with figures are the estimated growing time of columnar: ice crystals at water saturation and $1000 \mathrm{mb}$. 
position of the boundaries of various columnar ice crystals in the figures. As in Fig. 1 the hatched area is the region where rimed ice crystals were observed and depends markedly on the length of the minor axis as previously deduced (Ono, 1969). The times taken for crystals to grow to various sizes estimated from the crystal growth rate by vapor diffusion at water saturation (Ono, 1970) are marked in Fig. 2, showing that the riming on columnar ice crystals will commence after 8 to 10 minutes of growth.

\section{Evidence of collection of ice crystals near their place of origin}

Very small regular ice crystals, the examples of which are shown in Fig. 3, were often observed during their passages through clouds. As shown in Fig. 1 there is no discontinuity in the crystal size distribution, and so it is necessary to define a certain criterion for the very small regular ice crystals. In the present paper we define an ice crystal less than $100 \mu$ in the major axis which grows within about 2 mirutes at water saturation as the "small regular ice crystal". The fall velocities of thus defined small regular ice crystals are less than $10 \mathrm{~cm} \mathrm{sec}$ (Jayaweera and Cottis, 1969). As seen from Fig. 1 and Fig. 2 the axial ratio of the small regular ice crystal has slightly different values depending upon growth temperatures. The axial ratio $(\mathrm{a} / \mathrm{c})$ for needle-type crystals is 0.2 to 0.3 . It is 0.2 to 0.5 for sheath-type crystals and 0.3 to 0.6 for the solid/hollow-type columnar crystals as summarized in Table 2.

However, because of their smallness in size some doubt may remain as to whether the small regular ice crystals are to be attributed to any ice dislodged from the fuselage or the decelerator, or to spurious ice crystals grown during replications.

An argument against the dislodged frost possibility is that it seems very unlikely that the crystal form could have become as regular as those shown in Fig. 3 in the very short time (less than $0.1 \mathrm{sec}$ ) taken for ice to travel between the decelerator or the fuselage and the sampler. Crystals can form during replication if proper precautions are not taken,

Table 2. Axial ratios of small regular columnar crystals

\begin{tabular}{l|c|c|c|c}
\hline \multirow{2}{*}{} & \multicolumn{4}{|c}{ Shape of ice crystals } \\
\cline { 2 - 5 } & Solid/hollow & Needle & Sheath & Solid/hollow \\
\hline $\begin{array}{l}\text { Observed temperature }\left({ }^{\circ} \mathrm{C}\right) \\
\text { Axial ratio }(\mathrm{a} / \mathrm{c})\end{array}$ & -3.5 & -4 to -6 & -6 to -8 & -8 to -9.5 \\
\hline
\end{tabular}

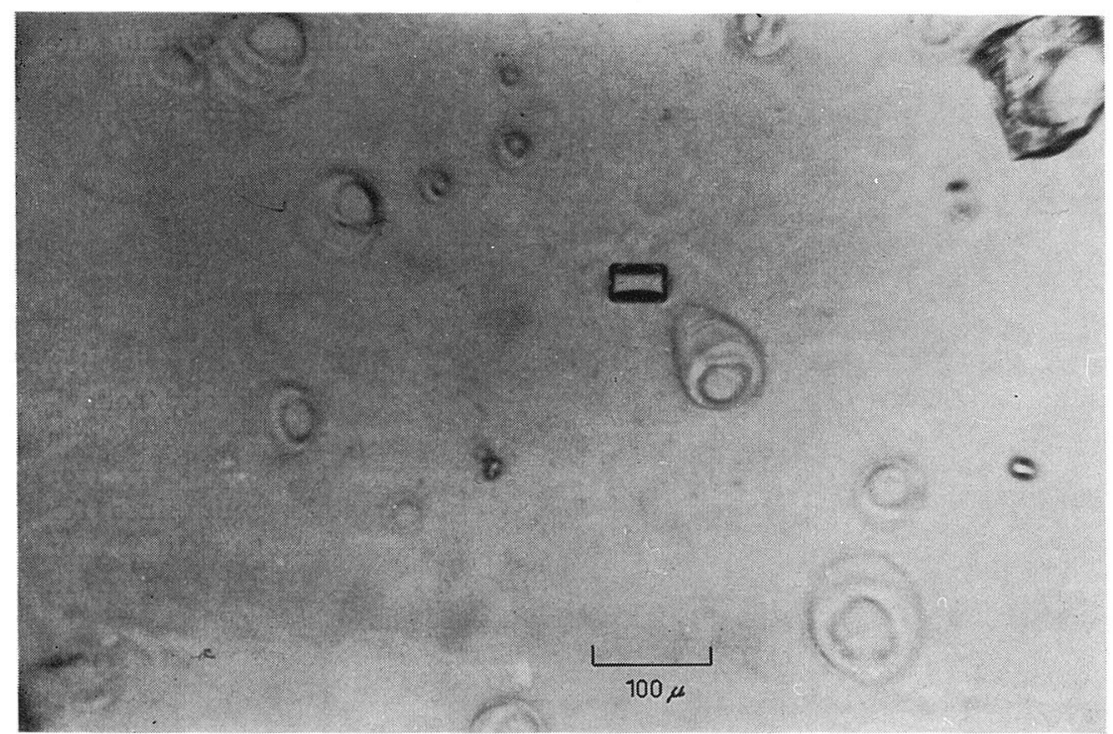

Fig. 3 Replicas of the small regular ice crystals 


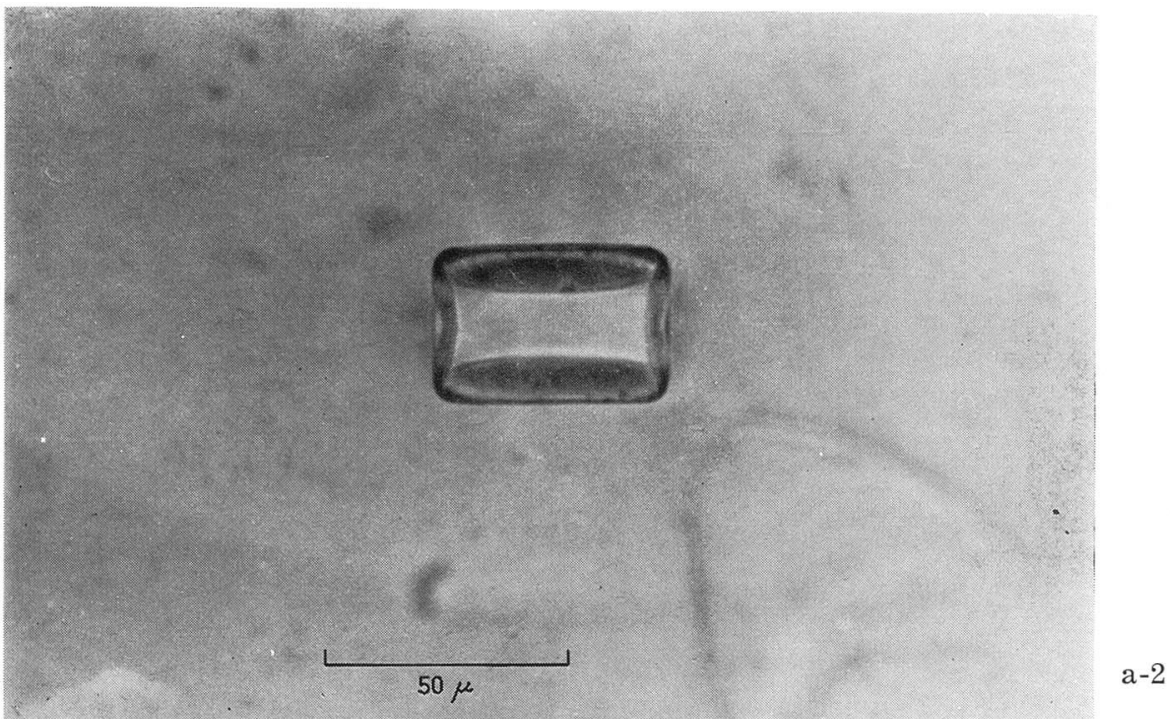

Fig. 3 Replicas of the small regular ice crystals (a) Observed at $-3.4 \mathrm{C}$ in Cloud No. 16, May 271968.
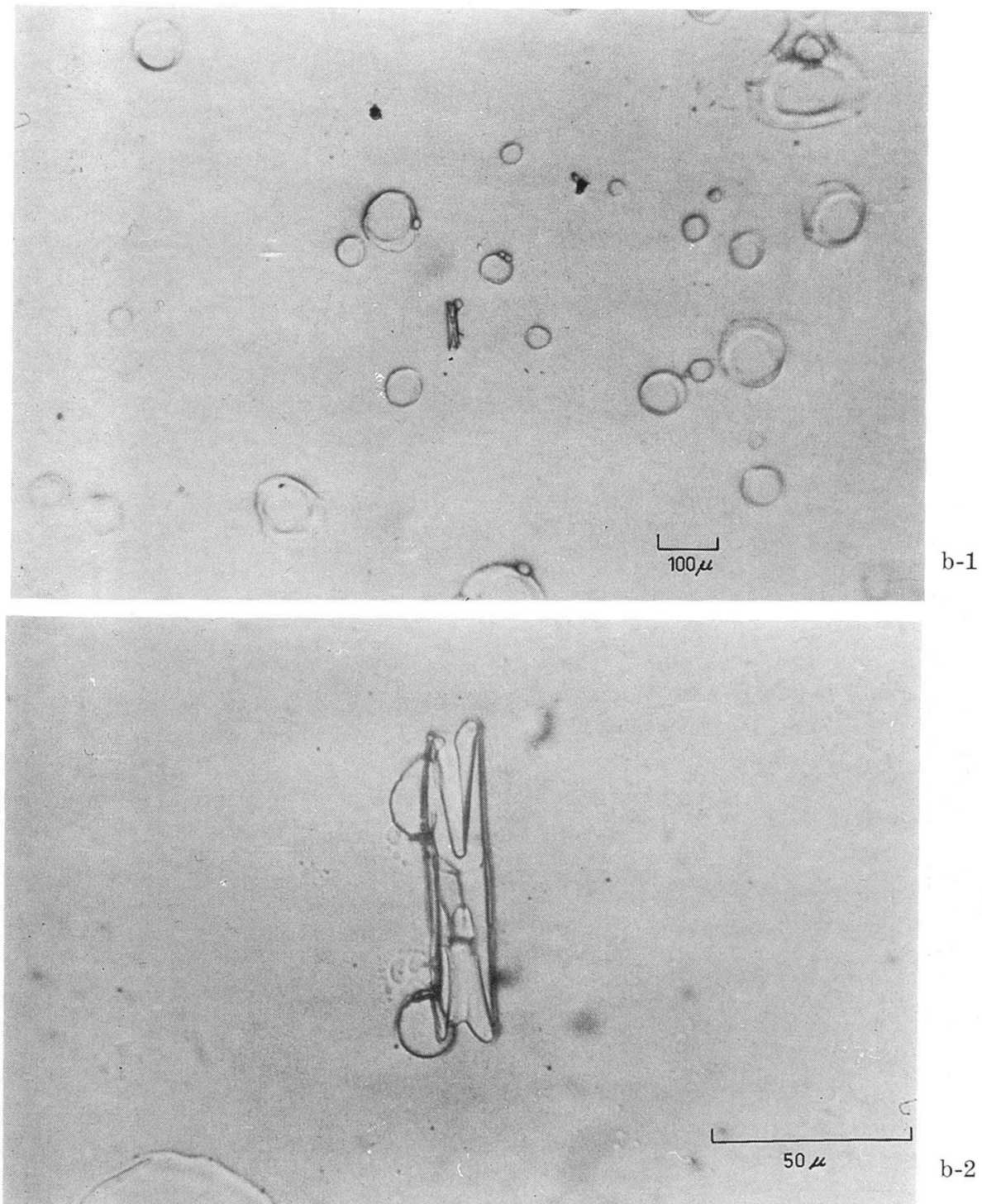

Fig. 3 Replicas of the small regular ice crystals (b) Observed at $-5.4 \mathrm{C}$ in Cloud No. 17, May 271968. 

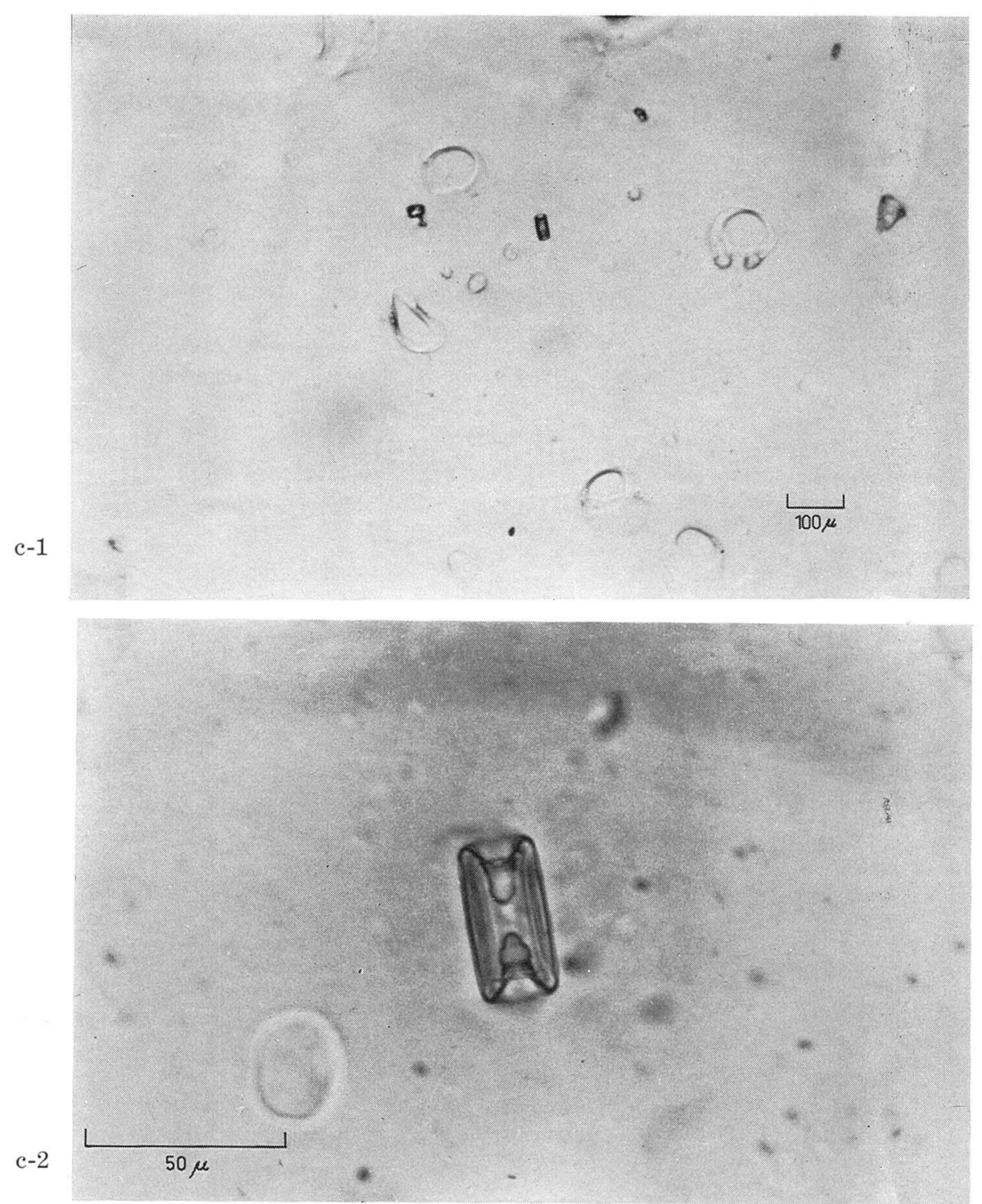

Fig. 3 Replicas of the small regular ice crystals (c) Observed at $-6.5 \mathrm{C}$ in Cloud No. 10, May 271968.

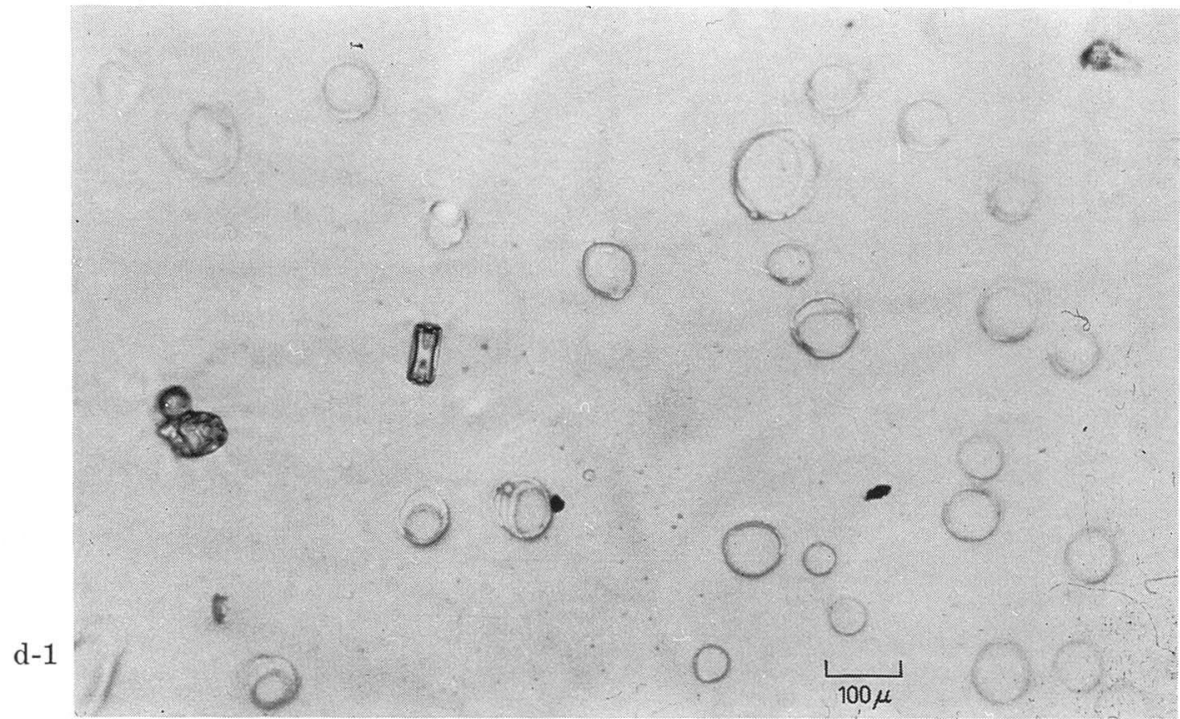




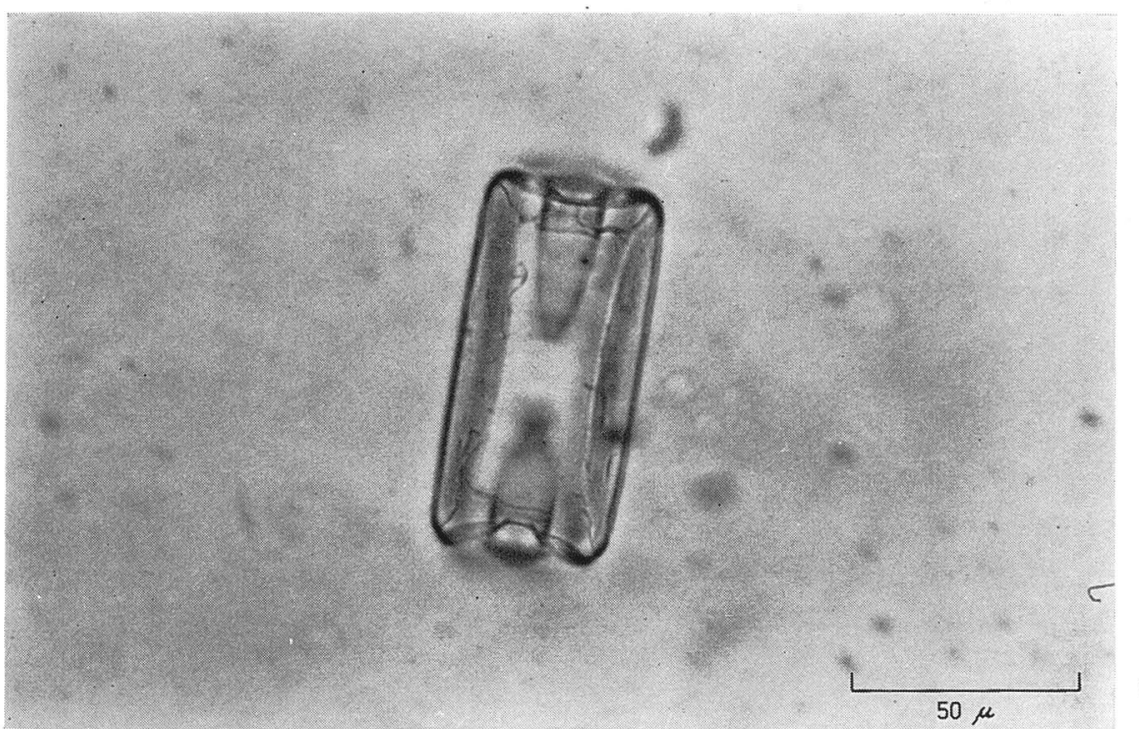

Fig. 3 Replicas of the small regular ice crystals (d) Observed at $-8.5 \mathrm{C}$ in Cloud No. 1-b, May 261968.

Also clearly shown are the replicas of drops up to $100 \mu$ in diameter.

but following Kobayashi (1955) the replicating solution was completely dehydrated with phosphorus pentoxide. In any case, as Kobayashi (1955) and later Sakurai (1968) have shown, spurios crystals have quite a different shape from those which we are considering.

We can, therefore, assume that these small regular ice crystals were originated in the cloud near or rather below the sampling level, considering the moderate updraft in clouds. The conditions existing in clouds would then be relevant to their manner of formation.

\section{5, Concentration of small regular ice crystals}

Could these newly formed small regular ice crystals have originated from the ice nuclei present in the atmosphere?

Estimations of ice nuclei concentrations in Australian conditions at humidities similar to those in a natural cloud have been made by Bigg (private communication) using $300 \mathrm{~L}$ slow expansion cloud chamber. The typical nucleus concentrations in relatively clean air

Fig. 4 Measured concentration of the small regular ice crystals in maritime cumulus clouds plotted against temperatures at the sampling level. The typical nucleus concentrations in Australian conditions are about $1 / \mathrm{m}^{3}$ activate at $-7 \mathrm{C}, 10 / \mathrm{m}^{3}$ at $-10 \mathrm{C}$ and $1 / \mathrm{L}$ at $-20 \mathrm{C}$ after Bigg (Private communication).

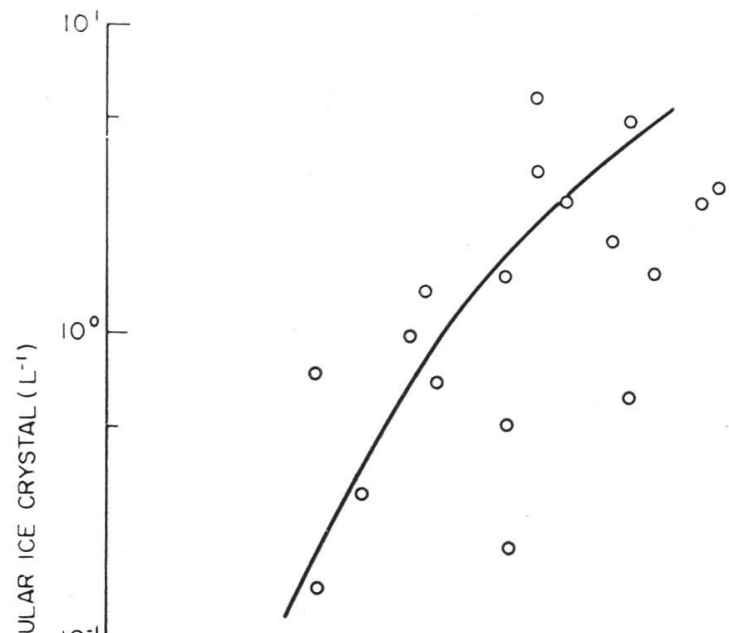
SPECTRUM(Bigg)

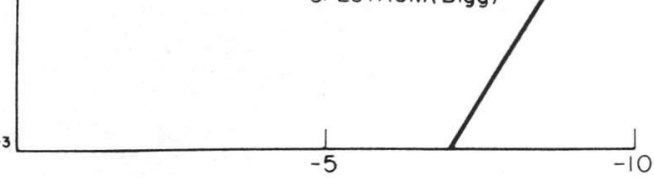

TEMPERATURE AT SAMPLING LEVEL $\left({ }^{\circ} \mathrm{C}\right)$ 
are about $1 / \mathrm{m}^{3}$ activated at $-7 \mathrm{C}, 10 / \mathrm{m}^{3}$ at $-10 \mathrm{C}$ and 1 /litre at $-20 \mathrm{C}$. (see Fig. 4)

In Fig. 4 the concentration of small regular ice crystals found in natural clouds is plotted as a function of temperatures at the sampling level and can be seen to be much higher than those of natural ice nuclei. The measurements were based on the replicas of intact ice crystals obtained from the decelerator making an allowance of a factor of 2 for uncertainty in the volume of air effectively sampled (Mossop et al., 1970), but no correction for the collection efficiency of the sampling device. The measured crystal concentrations may, therefore, have been underestimated. The temperatures at the sampling level was measured to an accuracy of $\pm 1 \mathrm{C}$.

A necessary conclusion, therefore, seems to be that the crystals could not have originated from ice nuclei present in the air, suggesting that these small regular ice crystals are formed as secondary products of some active ice crystal enhancement processes.

Although most of our cloud traverses were single 200 or $300 \mathrm{~m}$ below cloud summit, successive traverses of the same cloud were, on occasion, made from higher to lower levels. Murgatroyd and Garrod (1960) found a general tendency for a higher portion of a cloud to contain higher concentrations of ice crystals. Our traverses reveal a similar tendency but the highest crystal concentrations were not always confined to near the cloud summit.

\section{Microphysical conditions associated with the formation of samll regular ice crystals}

The clouds studied all had broad droplet spectra typical of maritime cumulus clouds of moderate size and updraft velocities (Mossop et al., 1970). As clearly shown in Fig. 3, drops of up to $100 \mu$ in diameter were replicated as well as the small regular ice crystal. There was no noticeable difference in droplet spectra between those with and without ice crystals, providing that the crystals were not numerous.

As summarized by Mossop et al. (1970) the glaciation processes in individual clouds are in recognisably different stages of microphysical development at the time of cloud penetration. Four stages of microphysical develop- ment can be distinguished:

(a) All-water clouds.

(b) Clouds containing water drops plus a small concentration of ice crystals relatively small in size.

(c) Clouds containing considerably less liquid water but an appreciable concentration of ice crystals, rimed ice crystals and graupel particles.

(d) Clouds from which the liquid water has almost entirely disappeared and in which the solid particles consist almost entierly of regular ice crystals and a few graupel particles.

It is in clouds of stage (c) that we most often observed small regular ice crystals. The glaciation in clouds of this stage has progressed considerably and the external appearance is less solid and becomes fuzzy as glaciation proceeds.

Thus the microphysical condition of the cloud suggests strongly that the formation of small regular ice crystals is associated closely with the riming process of ice crystal or graupel particle formation.

Does ice crystal enhancement depend upon riming on ice crystals? If so, we would expect that the higher the concentration of rimed crystals the higher would be the total

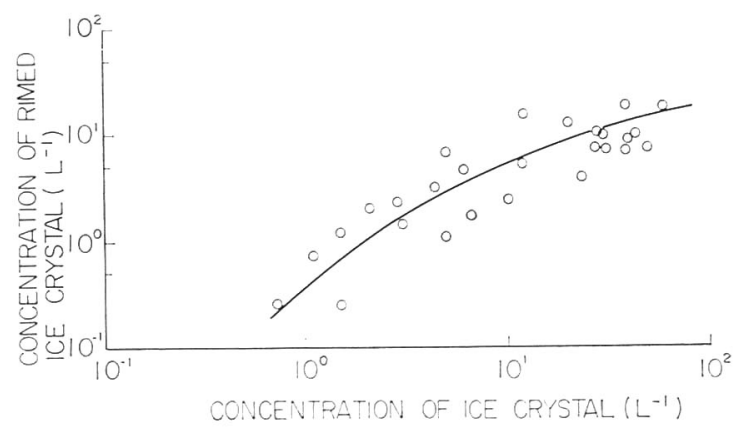

Fig. 5 Concentration of ice crystais iri maritime cumulus clouds plotted against concentrations of rimed ice crystals.

crystal concentrations. Fig. 5 is certainly consistent with this supposition.

Mention must here be made of the frozen droplets which were observed on the replicating slides. Most of the frozen droplets were formed in two-dimensional clusters associated with ice crystals or ice particles and the individual frozen droplet was observed less 


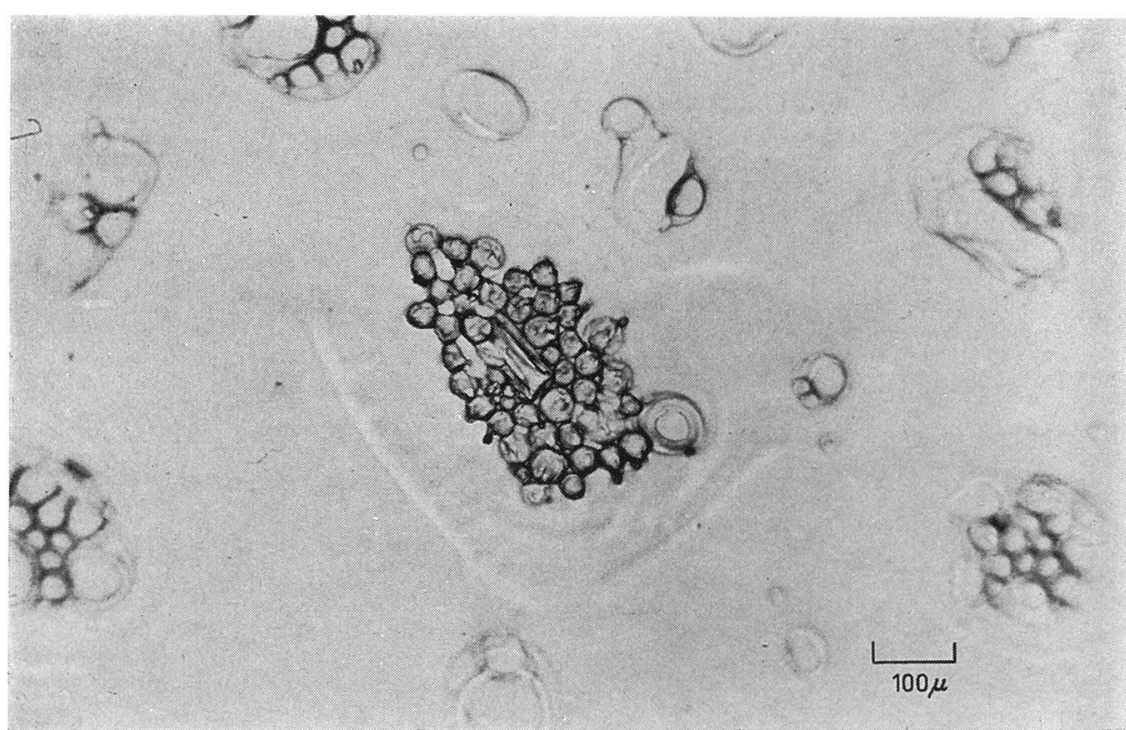

Fig. 6 Replicas of frozen drops (a) Cluster of frozen drops associated with ice particles observed at $-8.5 \mathrm{C}$ in Cloud No. 13-a, May 261968.

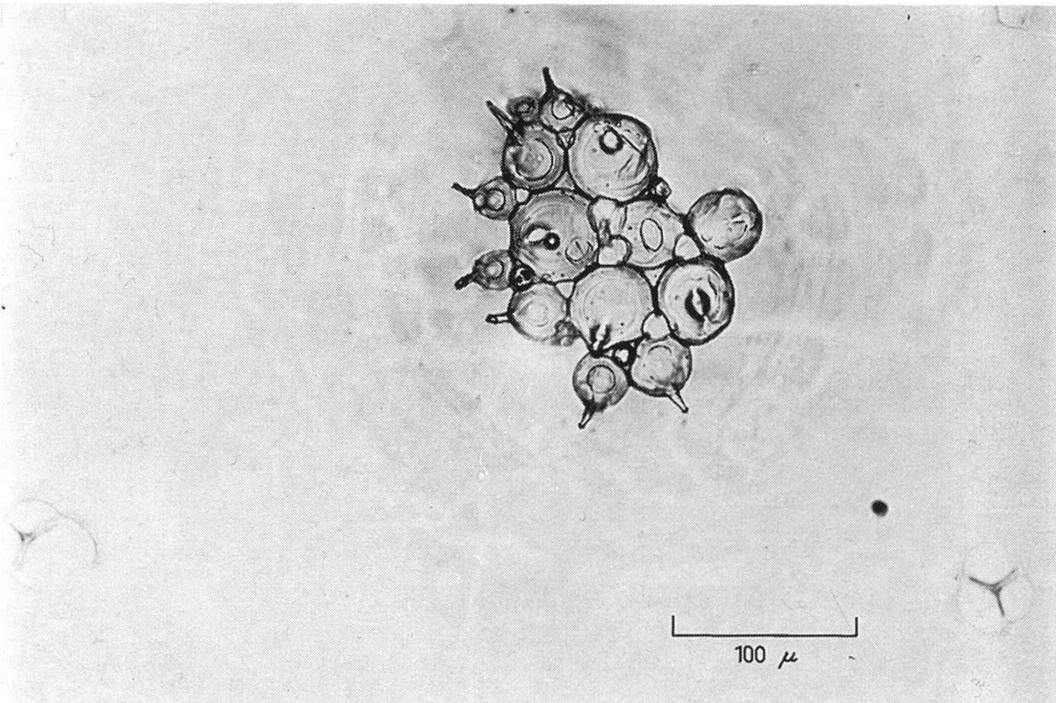

Fig. 6 Replicas of frozen drops (b) Graupel-like frozen drops observed at $-3.4 \mathrm{C}$ in Cloud No. 5, May 27 1968. The individual frozen drop has a spike extending outward in the radial direction.

frequently in clouds with summit temperatures colder than --8C. (see Fig. 6) We are not quite sure at present that these individual frozen droplets were formed in the air or formed in the process of sampling and replication. However, as will be described later, the probability of ice nuclei or ice crystals colliding with water drops and freezing them seems too small to make any significant contribution to the formation of frozen droplets at tempera- tures above $-10 \mathrm{C}$. Therefore, it may be supposed that these frozen droplets are to be attributed either to the impaction on replicating slides with such high velocity as more than $15 \mathrm{~m} \mathrm{sec} \mathrm{ser}^{-1}$ or to the interaction between water drops and the sampling device.

In summary, circumstantial evidence indicates that the main multiplication of ice crystals appears to proceed simultaneously with the riming process. When ice crystals 
grow large enough to accrete drops, fragments of ice may be thrown off as water drops freeze. These ice fragments would be unable to take part in any collision growth until they had first grown considerably from vapor phase into regular ice crystals. Ice crystals originated from fragments would then grow into crystals large enough to accrete supercooled water drops in their turn and the process would be repeated.

\section{Discussion}

Let us consider the possible mechanisms of ice crystal multiplication that have hitherto been suggested and ask which appears to be consistent with the observations that have been described above.

(a) Freezing of water drops nucleated by collision with ice crystals

When a water drop freezes by internal nucleation or through collision with ice crystals, it will under certain conditions throw off ice splinters or ice fragments. This has been corroborated in laboratory experiments by Johnson and Hallett (1968), Dye and Hobbs (1968), Brownscombe and Thorndike (1968) and recently Takahashi and Yamashita (1970).

More relevant to the present discussions were the observations of the University of Chicago's project “White top” summarized by Braham (1964). Braham reported that ice particles were observed in most of the convective summer clouds in Missouri by the time when cloud tops reached a temperature of about $-10 \mathrm{C}$ and ice pellets were the dominant solid hydrometeors. They supposed that those ice pellets were formed as a result of the freezing of large water drops in the air and postulated that the shattering or splintering of large water drops was the source of large concentrations of ice particles observed in clouds.

According to their postulation (Koenig, 1966), the numerous ice fragments thrown off in the final stage of drop freezing would be small, say a few microns in size, and therefore would be unable to take part in any collision growth process until they had first grown considerably from vapor phase. Thus from their postulation one might expect numerous regular-shaped ice crystals of various sizes in clouds as observed in the present cases. However, contrary to this expectation, their observations showed that the irregular ice pellets were the dominant solid hydrometeors.

Another and more serious drawback in their interpretation of the observational results is that it is left unexplained how the freezing of water drops occurred in such a warm cloud with summit temperature above $-10 \mathrm{C}$

Vali (1968) has discussed the freezing of raindrop-sized samples of rain water at some length and has shown that there is an appreciable probability that a drop will freeze at temperature as warm as $-5 \mathrm{C}$ by internal

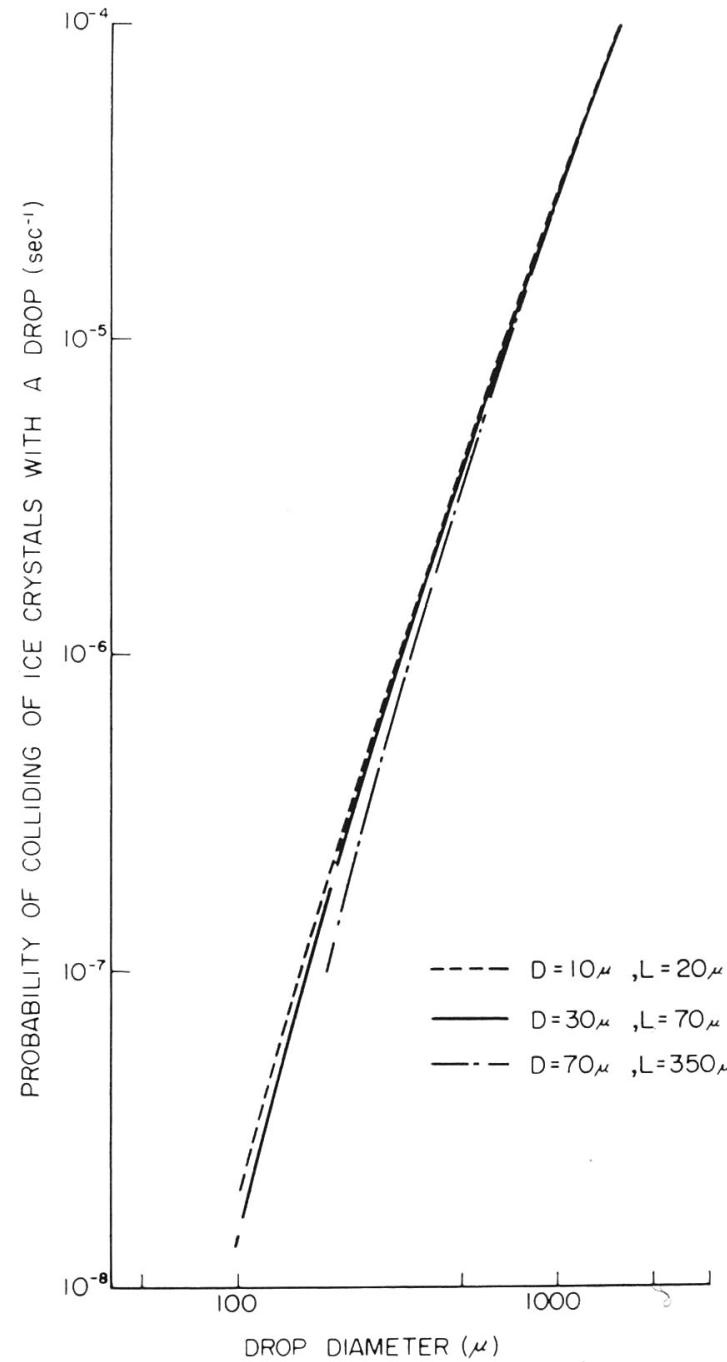

Fig. 7 Probability of colliding of ice crystals with a water drop. The concentration of ice crystals $10^{-5} / \mathrm{cm}^{3}$ is equal to the concentration of ice nuclei activated at $-10 \mathrm{C}$. 
nucleation. However, this depends critically on how much air the growing drop has effectively depleted of ice nuclei and their concentrations. Since Vali has evidence that the nuclei are extremely small in size this volume may be a good deal larger than one might expect.

Alternatively we might assume that freezing of large water drops takes place by collision with existing ice crystals. Fig. 7 shows the probability of collision calculated by assuming the collection efficiency of a water drop for ice crystals to be unity, and the concentration of ice crystals to be $10^{-5} \mathrm{~cm}^{-3}$, which is equal to that of ice nuclei activated at $-10 \mathrm{C}$ in Australian conditions.

It can be seen from Fig. 7 that the probability is not much different for three different sizes of ice crystals: $\mathrm{D}=10 \mu \mathrm{L}=20 \mu$, $\mathrm{D}=30 \mu \mathrm{L}=70 \mu, \mathrm{D}=70 \mu \mathrm{L}=350 \mu$. The probability is about $10^{-8} \mathrm{sec}^{-1}$ for a drop of $100 \mu$ in diameter. It is $5 \times 10^{-6} \mathrm{sec}^{-1}$ for a $500 \mu$ drop and less than $3 \times 10^{-5} \mathrm{sec}^{-1}$ for a drop of $1000 \mu$.

The estimated values are larger than the probability of ice nuclei colliding with a water drop by Brawnian motion (Takeda, 1968) or the probability of spontaneous nucleation at temperatures above-10C. However, it is still not large enough to make any significant contribution to ice phase development in clouds of summit temperatures warmer than $-10 \mathrm{C}$.

The probability of ice crystals colliding with a water drop is very much dependent on the concentration of existing ice crystals and if the concentration of ice crystals is of the order of $100 \mathrm{~L}^{-1}$, then the probability becomes significantly large. From our observations, however, it is very rare to see many supercooled water drops in clouds with a crystal concentration as large as $100 \mathrm{~L}^{-1}$.

(b) Accretion-freezing process (riming process)

It was first reported by Findeisen (1940) that ice splinters were thrown off when a supercooled water drop of $40 \mu$ in diameter were impacted on a growing ice deposit. Since then many authors (Mason and Maybank, 1960; Latham and Mason, 1961; and Johnson and Hallett, 1968) have observed that under certain conditions individual drops freezing on accretion can throw off pieces of ice in the final stage of the freezing.

Since rimed ice crystals and graupel pellets were common in clouds where we observed many small regular ice crystals, this riming process is one which requires careful examinations. Will sufficient accretion occur between the rare initial ice crystals and water droplets to explain the ice crystal enhancement within a reasonable space of time? How many splinters must we postulate for the measured concentration to be attained within this time?

Fig. 8 shows the resulting probability of accretion of water droplets on ice crystals of five different sizes. In the calculation we assume a drop spectrum typical of maritime cumuli (Squires, 1958) and a collection efficiency of columnar ice crystals for water droplets based on the results of the author (Ono,

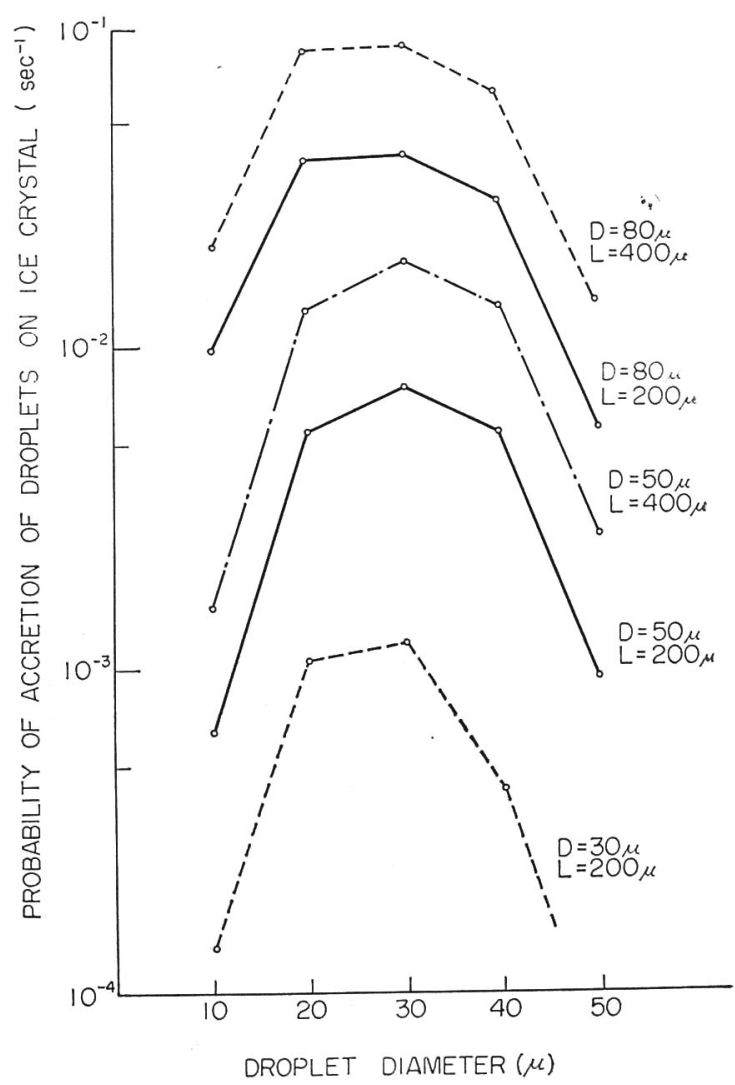

Fig. 8 Probability of accretion of water drops on an ice crystal. The concentration of water drops is based on the results by Squires (1959) obtained in maritime cumuli. It is $3 / \mathrm{cm}^{3}$ for drops of $10 \mu$ in diameter, $8.5 / \mathrm{cm}^{3}$ for $20 \mu$ drops, $9 / \mathrm{cm}^{3}$ for $30 \mu$ drops, $6.4 / \mathrm{cm}^{3}$ for $40 \mu$ drops and $1.4 / \mathrm{cm}^{3}$ for $50 \mu$ drops. 
1970).

The probability of accretion of water droplets on an ice crystal of $\mathrm{D}=80 \mu \quad \mathrm{L}=400 \mu$ is about $10^{-1} \mathrm{sec}^{-1}$. It is about $2 \times 10^{-2} \mathrm{sec}^{-1}$ for an ice crystal of $\mathrm{D}=50 \mu \mathrm{L}=400 \mu$ and about $10^{-3} \mathrm{sec}^{-1}$ for an ice crystal of $\mathrm{D}=30 \mu \mathrm{L}=200 \mu$. As expected from the observations the probability of accretion of water droplets on ice crystals is significantly larger than that of collision of ice crystals with a water drop. This is mainly because of the higher concentration of water drops in clouds compared to that of ice crystals initially formed on ice nuclei in the air.

The estimated probabilities agree well with the onset of riming (see Fig. 2). The relatively large probabilities for water drops of 20 to $40 \mu$ in diameter shown in this figure also agree with the observations, the size distribution of accreted water droplets having a peak at the diameter of about 30 to $40 \mu$.

(c) Efficiency of the ice crystal multiplication by accretion-freezing process

The time involved then depends critically on the mean number of splinters per drop.

Let us calculate the time taken for ice crystal concentrations to increase by a factor of 10 under the following assumptions;

(1) Ice crystals $(\mathrm{D}=80 \mu \mathrm{L}=200 \mu)$ formed initially on ice nuclei of $10^{-5} \mathrm{~cm}^{-3}$ collide with $1 \mathrm{~mm}$ diameter drops of $50 \mathrm{~m}^{-3}$ (Koenig, 1966), causing the drops to freeze and throw off ice splinters.

(2) Ice crystals $(\mathrm{D}=80 \mu \mathrm{L}=200 \mu)$ formed initially on ice nuclei of $10^{-5} \mathrm{~cm}^{-8}$ accrete $30 \mu$ water droplets of $9 \mathrm{~cm}^{-3}$, causing the droplets to freeze and throw off ice pllinters. In each case let us assume $\mathrm{s}=0.1,1$ and 10 splinters per frozen drop.

Table 3 shows the time required to increase the concentration of ice crystals by a factor of 10. This makes it quite evident that unless the splinter production is vastly greater for large drops than for small ones, only the accretion-freezing process has the chance of being important.
As shown in Fig. 2 the growing time of the ice crystal of $\mathrm{D}=80 \mu \mathrm{L}=200 \mu$ is about 10 minutes; then in the case of the accretionfreezing process the total time required to increase the crystal concentration by a factor of 10 is about 50 minutes for $s=0.1$. It is about 14 minutes for $\mathrm{s}=1$ and 11 minutes for $\mathrm{s}=10$.

The observed rate of crystal multiplication in a similar type of clouds (Mossop et al., 1970) is about a factor of 10 in about 10 minutes. So that the table shows a splinter production of the order of several ice fragments per drop is necessary for this mechanism to work.

The most widely quoted results on the production rate of ice splinters are probably those of Latham and Mason (1961), who observed copious ice splinters produced during riming and growth of soft hail in the laboratory conditions. They have shown that droplets of diameters less than $30 \mu$ produced few ice splinters and the rate of splinter production increased with increasing drop diameter until with large drops moving at high fall velocities, splashing occurred.

However, many authors who have made laboratory experiments similar to those of Latham and Mason have so far failed to reproduced their results from the measurements of the charge acquired by the ice surface and, therefore, cast a doubt upon Latham and Mason's observations.

Recent results by Johnson and Hallett (1968) have shown that the symmetrical heat transfer from freezing drops is the most essential factor controlling the fragmentation of freezing drops. In relation to this point they state that drops larger than $10 \mu$ in diameter are unlikely to fracture on freezing in free fall in the atmosphere but that drops freezing on accretion on ice crystals provide more likely physical situation for ice fragmentation under certain conditions.

In summary, from the numerical estimation we come to the conclusion that ice splinter

Table 3. Time required to increase the crystal concentration by a factor of 10 ( $\mathrm{sec}$ )

\begin{tabular}{l|c|r|r}
\hline \hline & \multicolumn{3}{|c}{ Rate of splinter production per drop } \\
& 0.1 & 1 & 10 \\
\hline Accretion-freezing process & $2.5 \times 10^{3}$ & $2.5 \times 10^{2}$ & $2.5 \times 10^{1}$ \\
Collision-freezing process & $5 \times 10^{5}$ & $5 \times 10^{4}$ & $5 \times 10^{3}$ \\
\hline
\end{tabular}


production by freezing of accreted water drops on ice crystals is a more likely process of crystal multiplication in maritime convective clouds and it is suggested that the production rate of ice splinters should be of the order of several splinters per accreted drop.

\section{Conclusion}

The most important result of this work is that for maritime cumulus clouds with summit temperatures warmer than $-10 \mathrm{C}$, the small regular ice crystals were found to form preferentially in clouds where water drops and ice crystals grown large enough for the onset of riming co-exist. Their concentration was 2 or 3 orders of magnitude larger than those of ice nuclei present in the air.

This narrows down the range of possible crystal multiplication mechanisms. For the broad size distribution of ice crystals rules out the accumulation zone hypothesis, and then we are left with the possibilities of splintering of large water drops either nucleated by capture of an ice crystal or upon being accreted and frozen by ice crystals.

Unless the large drops are far more efficient splinter producers than laboratory experiments suggested, the only mechanism so far considered that seems at all reasonable is splintering during riming on ice crystals. Laboratory experiments cannot yet tell whether the requisite several splinters per drop is reasonable.

At least one thing is certain-it is not appropriate in clouds of this sort to suppose that the concentration of ice nuclei in the air will be adequate to determine the concentration of ice crystals existing in clouds.

\section{Acknowledgement}

The main body of the present research was accomplished during the author's stay as a visiting research fellow at the Division of Radiophysics, C.S.I.R.O., on leave from the Geophysical Institute, Tokyo University. The study and preparation of the paper was later continued at the Meteorological Research Institute, Tokyo, Japan. I am indebted to Drs. E.G. Bowen, S.C. Mossop, E.K. Bigg, J.L. Brownscombe and Mr. J. Warner of the division for their encouragements, helpful suggestions and stimulating discussions. The field experiments profited by the generous coopera- tion of many individuals, and I would like to thank the late Mr. K.J. Heffernan, Mr. E.R. Wishart, Mr. R.E. Cottis and Mr. H. Flood for their skillful technical assistance.

\section{References}

Braham, R. R. Jr., 1964: What is the role of ice in summer rain showers? J. Atmos. Sci., 21, 640-645.

Brownscombe, J. L. and J. Hallett, 1967: Experimental and field studies of precipitation particles formed by the freezing of supercooled water. Quart. J. Roy. meteor. Soc., 93, 455473.

and N. S. C. Thorndike, 1968: Freezing and shattering of water drops in free fall. Nature, 220, 687-689.

Dye, J. E. and P. V. Hobbs, 1968: The influence of environmental parameters on freezing and fragmentation of suspended water drops. J. Atmos. Sci., 25, 82-96.

Findeisen, W., 1940: On the origin of lightning electricity. Meteor. Z., 57, 201-221.

Hobbs, P. V. and A. J. Alkezweeny, 1968: The fragmentation of freezing water droplets in the free fall. J. Atmos. Sci., 25, 881-888.

Jayaweera, K. O. L. F. and R. E. Cottis, 1969: Fall velocity of plate-like and columnar ice crystals. Quart. J. Roy. meteor. Soc., 95, 703709 .

Johnson, D. A. and J. Hallett, 1968: Freezing and shattering of supercooled water drops. Quart. J. Roy. meteor. Soc., 94, 468-482.

Kobayashi, T., 1955: Studies on small ice crystals III, some remarks on replica methods. Contri. Inst. Low Temp. Sci. 8, 75-86.

Koenig, L. R., 1966: Numerical test of the validity of the drop-freezing splintering hypothesis of cloud glaciation J. Atmos. Sci., 23, 726740 .

— 1968: Some observations suggesting ice multiplication in the atmosphere. J. Atmos. Sci., 25, 460-163.

Latham, J. and B. J. Mason, 1961: Generation of electric charge associated with the formation of soft hail in thunderclouds. Proc. Roy. Soc. A, 260, 537-549.

Mason, B. J., 1956: The nucleation of supercooled water clouds. Sci. Progress, 44, 479-499.

and J. Maybank, 1960: The fragmentation and electrification of freezing water drops. Quart. J. Roy. meteor. Soc., 86, 176188.

Mossop, S.C. and A. Ono, 1969: Measurements of ice crystal concentration in clouds. J. Atmos. Sci., 26, 130-137.

, - and E. R. Wishart, 1970: Ice par- 
ticles in maritime clouds near Tasmania. Quart. J. Roy. meteor. Soc., 96, 487-508.

Murgatroyd, R. J. and M. P. Garrod, 1960: Observations of precipitation elements in cumulus clouds. Quart. J. Roy. meteor. Soc., 86, 167-175.

Ono, A., 1969: The shape and riming properties of ice crystals in natural clouds. J. Atmos. Sci., 26, 138-147.

1970: Growth mode of ice crystals in natural clouds. J. Atmos. Sci. 27, 649-658.

Sakurai, K., 1968: On long prismatic ice crystals formed in replica solution. J. meteor. Soc. Japan, 46, 150-151.

Squires, P., 1958: The microstructure and collo- idal stability of warm clouds. Part I. The relation between structure and stability. Tellus, 10, 256-261.

Takahashi, T. and A. Yamashita, 1970: Shattering of frozen water drops in a supercooled cloud. J. meteor. Soc. Japan, 48, 373-376.

Takeda, T., 1968: Solid precipitation in supercooled clouds. Part I. Freezing of a supercooled water drop due to collision of ice crystals. J. meteor. Soc. Japan, 46, 14-28.

Vali, G., 1968: The freezing-nucleus content of precipitation and its relation to the formation of ice in the clouds. Proc. Intern. Conf. Cloud Physics, Tronto, 232-237. 